\title{
Ishak Score 3
}

National Cancer Institute

\section{Source}

National Cancer Institute. Ishak Score 3. NCI Thesaurus. Code C95153.

Fibrosis extends beyond the portal areas. Occasional portal to portal bridging fibrosis is present. 